\section{ORIGINAL RESEARCH}

W. Shirai
S. Ito
T. Hattori

\title{
Linear T2 Hyperintensity along the Medial Margin of the Globus Pallidus in Patients with Machado- Joseph Disease and Parkinson Disease, and in Healthy Subjects
}

BACKGROUND AND PURPOSE: Linear hyperintensity along the medial margin of the internal segment of the globus pallidus (GPi) on T2-weighted images from patients with Machado-Joseph disease (MJD) was recently reported. In this study, we aimed to compare the prevalence of this linear hyperintensity between patients with MJD and patients with Parkinson disease, and in control subjects.

\begin{abstract}
MATERIALS AND METHODS: We report the prevalence of linear hyperintensity along the GPi in 10 consecutive patients with MJD, 38 with Parkinson disease (PD), and 25 control subjects. We evaluated the prevalence of linear hyperintensities on T2-weighted axial images in these groups, and in nearly age-matched subjects younger than 60 years. We performed statistical analysis using the $\chi^{2}$ test and Fisher exact test to compare the prevalence of the hyperintensity in each group.
\end{abstract}

\begin{abstract}
RESULTS: The prevalence of the linear hyperintensity in patients with MJD was significantly higher than that in patients with PD and in control subjects ( $P=.014$ and .006 , respectively). In subjects younger than 60 years, the prevalence of the hyperintensity in patients with MJD was significantly higher than that in patients with $\mathrm{PD}$ and control subjects $(P=.011$ and $P<.001$, respectively). In the control subjects, the linear hyperintensity tended to be greater in subjects older than 60 years (8/14 [57\%] patients) than in subjects younger than 60 years (2/11 [18\%] patients; $P=.058$ ). In patients with $\mathrm{PD}$, the prevalence did not differ between patients older than 60 years and patients younger than 60 years.
\end{abstract}

CONCLUSION: Linear hyperintensity along the globus pallidus is frequently found in patients with MJD, but it can be also found in patients with PD and in control subjects, probably because of the normal aging process.

$\mathbf{M}$ achado-Joseph disease (MJD) is an autosomal dominant neurodegenerative disease caused by the expansion of CAG trinucleotide repeats in a gene located at chromosome 14q. ${ }^{1} \mathrm{MJD}$ is clinically characterized by variable combinations of cerebellar ataxia, progressive external ophthalmoplegia, pyramidal signs, dystonia, muscular rigidity, amyotrophy, and peripheral neuropathy. Neuropathologic findings indicate that the spinocerebellar tracts are affected, along with the vestibular and dentate nuclei. There is also frequent involvement of the internal segment of the globus pallidus (GPi), the substantia nigra, and the subthalamopallidal connections. ${ }^{1}$

Recently, Yamada et $\mathrm{al}^{2}$ reported that T2-weighted images (T2WI) of patients with MJD frequently showed linear hyperintensity along the GPi. This finding is helpful for diagnosing MJD, and it is important because MJD is an inherited, slowly progressive disease, and early genetic counseling is usually recommended. Observations in our clinical practice are consistent with this finding, but we have also observed linear hyperintensity along the GPi in patients with other diseases, such as Parkinson disease (PD), and in elderly subjects. PD is a common disease that affects the nigrostriatal fibers, some parts of which run along the medial margin of the GPi.

Received February 27, 2007; accepted after revision April 23.

From the Department of Neurology, Graduate School of Medicine, Chiba University, Chiba, Japan.

Please address correspondence to Shoichi Ito, MD, Department of Neurology, Graduate School of Medicine, Chiba University, 1-8-one Inohana, Chuo-ku, Chiba, 260-8670, Japan; e-mail: sito@faculty.chiba-u.jp

DOI 10.3174/ajnr.A0705
Here, we investigated the presence of linear hyperintensity on T2WI along the medial margin of the GPi in patients with MJD or PD and in healthy subjects to better understand its clinical significance.

\section{Materials and Methods}

There were 10 consecutive patients with MJD, 38 with PD, and 25 control subjects who were enrolled in our study. We confirmed the diagnosis of MJD by measuring the expansion of the CAG repeat of the MJD1 gene. ${ }^{3}$ Clinical diagnosis of PD was made in accordance with the UK Parkinson's Disease Society Brain Bank Clinical Diagnostic Criteria. ${ }^{4}$ The control subjects were patients without any neurologic abnormalities who complained of headache or dizziness. Demographic data on the subjects are listed in the Table.

Brain MR imaging was performed with a 1.5T Signa Horizon MR scanner (GE Healthcare, Milwaukee, Wis). We acquired T2WI using the following parameters: fast spin-echo, TR, $4000 \mathrm{~ms}$; TE, $99 \mathrm{~ms}$; number of averages, 2; section thickness, $6.0 \mathrm{~mm}$; section gap, 1.5 $\mathrm{mm}$; FOV , $230 \times 230 \mathrm{~mm}$; and matrix, $256 \times 256$. The axial sections were set to lie parallel to the anteroposterior commissure line. The axial section with the largest GPi was selected for evaluation. This section always contained the posterior limbs of the internal capsule and the thalamic pulvinar that were usually rostral to the anterior commissure. On this selected section, 2 experienced neurologists (W.S. and S.I.) who were blinded to the diagnosis evaluated whether linear hyperintensity along the medial margins of the GPi was present or absent. We performed statistical analysis using the $\chi^{2}$ test and Fisher exact test. We calculated the Cohen $\kappa$ value as a marker representing interrater reliability. 


\begin{tabular}{|c|c|c|c|c|c|c|}
\hline \multicolumn{7}{|c|}{ Demographic data and scores of linear hyperintensity along the medial margin of the globus pallidus on T2-weighted axial images } \\
\hline & \multicolumn{3}{|c|}{ All Subjects } & \multicolumn{3}{|c|}{ Subjects Younger than 60 Years } \\
\hline & $\operatorname{MJD}(n=10)$ & $\mathrm{PD}(n=38)$ & $\mathrm{NC}(n=25)$ & $\operatorname{MJD}(n=9)$ & $\mathrm{PD}(n=6)$ & $\mathrm{NC}(n=11)$ \\
\hline Men/Women & $6 / 4$ & $23 / 15$ & $11 / 14$ & $6 / 3$ & $5 / 1$ & $4 / 7$ \\
\hline Age (y) & $45 \pm 10$ & $66 \pm 7$ & $60 \pm 11$ & $42 \pm 7$ & $53 \pm 3$ & $51 \pm 9$ \\
\hline Disease duration (y) & $12 \pm 7$ & $5 \pm 5$ & & $11 \pm 7$ & $4 \pm 3$ & \\
\hline \multicolumn{7}{|c|}{$\begin{array}{l}\text { Linear hyperintensity along the } \\
\text { globus pallidus }\end{array}$} \\
\hline Absent, $n(\%)$ & $0(0)$ & $15(39)$ & $12(48)$ & $0(0)$ & $4(67)$ & $9(82)$ \\
\hline Present, $n(\%)$ & $10(100)^{*}$ & $23(61)$ & $13(52)$ & $9(100)^{*}$ & $2(33)$ & $2(18)$ \\
\hline
\end{tabular}

Note:-MJD indicates Machado-Joseph disease; PD, Parkinson disease; NC, controls.
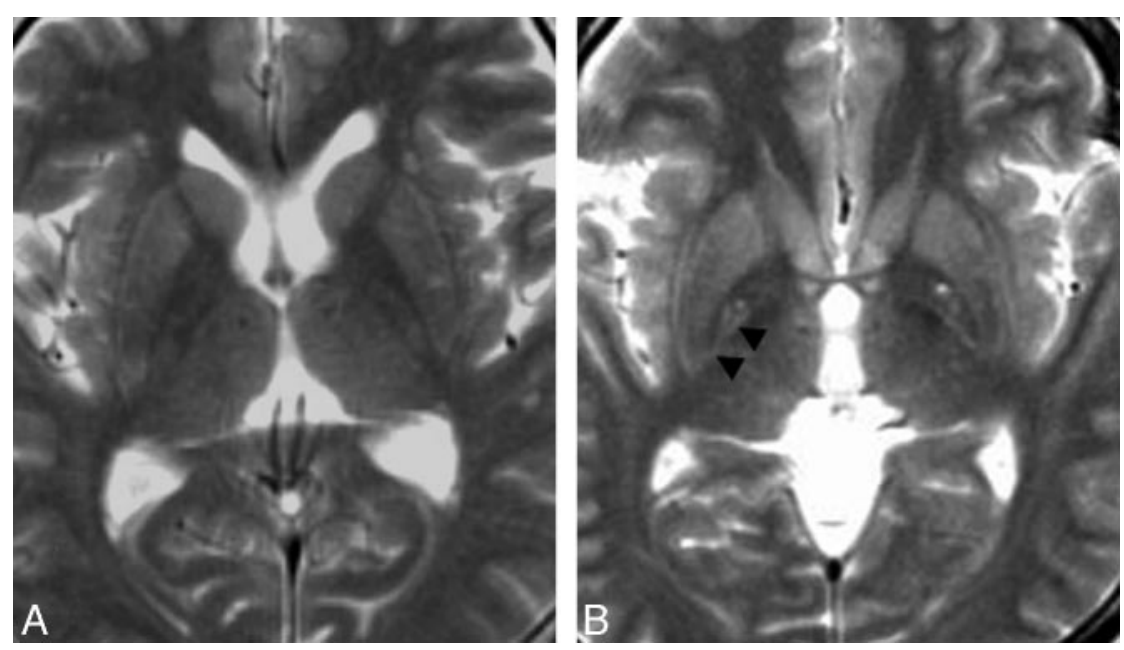

Fig 1. T2-weighted axial images in which the largest areas of the globus pallidus are shown. $A$, There is no apparent linear hyperintensity along the medial margin of the globus pallidus. $B$, There is apparent linear hyperintensity on both sides along the medial portion of the globus pallidus. These signals are hyperintense (ie, similar to the gray matter signals) and are located in the outer portion of the posterior limb of the right internal capsule. In addition, there are dilated perivascular spaces in the bilateral globus pallidi.

\section{Results}

All 10 of the patients with MJD had linear hyperintensity along the medial margin of the GPi (Table). Of the 38 patients with $\mathrm{PD}$, the linear hyperintensity was absent in 15 (39\%) patients and present in $23(61 \%)$ patients. Of the 6 patients younger than 60 years with PD, the hyperintensity was absent in 4 (67\%) and present in $2(33 \%)$. Of the 25 control subjects, the linear hyperintensity was absent in $12(48 \%)$ and present in 13 (52\%). Of the 11 control subjects younger than 60 years, the hyperintensity was absent in $9(82 \%)$ and present in $2(18 \%)$. Interrater reliability was moderate $(\kappa$ value $=0.47)$.

In the statistical analysis, the prevalence of the linear hyperintensity in patients with MJD was found to be significantly higher than that in the patients with $\mathrm{PD}$ and in the control subjects $(P=.014$ and $P=.006$, respectively). There was no significant difference between the patients with $\mathrm{PD}$ and the control subjects. In subjects younger than 60 years, the prevalence of the hyperintensity in patients with MJD was significantly higher than that in patients with PD or in the control subjects $(P=.011$ and $P<.001$, respectively). In the control subjects, linear hyperintensity had a tendency to be greater in subjects older than 60 years $(8 / 14[57 \%])$ than in subjects younger than 60 years $(2 / 11[18 \%] ; P=.058)$. In patients with $\mathrm{PD}$, the prevalence did not differ between patients older than 60 years and patients younger than 60 years.

\section{Discussion}

In our study, linear hyperintensity along the medial margin of the GPi on T2WI was frequently observed in patients with MJD, which is consistent with the results of a previous report ${ }^{2}$ in which hyperintensity along the GPi was considered to indi- cate the degeneration of the lenticular fasciculus, which originates from the inner portion of the GPi and runs dorsomedially through the fibers of the internal capsule. ${ }^{5,6}$ The GPi and its efferent fibers, such as the lenticular fasciculus and ansa lenticularis, are frequently degenerated in patients with MJD, and there is also frequent involvement of the nigrostriatal and subthalamopallidal connections, ${ }^{1,7}$ which also run along the $\mathrm{GPi}^{8,9}$ Thus, linear T2 hyperintensity in patients with MJD might reflect not only degeneration of the lenticular fasciculus, but also degeneration of other fibers near the GPi.

Control subjects older than 60 years had more frequent linear hyperintensity along the GPi than control subjects younger than 60 years. Thus, we hypothesized that linear hyperintensity along the GPi was related to normal aging of the brain, though we do not know what structural changes might be causing the linear hyperintensity. Hyperintensity can be interpreted as an abnormal sign when it appears in young subjects, especially in those younger than 60 years, but should probably be interpreted as a sign of normal aging of the brain when it appears in older subjects, especially in those older than 60 years, though no research supports this hypothesis. On the contrary, several reports show no apparent correlation between aging and the volume of the GPi. ${ }^{10-12}$ We speculate that the hyperintensity along the GPi does not necessarily correspond to atrophy of the GPi.

In patients with $\mathrm{PD}$, the score of the linear hyperintensity along the GPi was not significantly different from that in control subjects. Because most of the patients with PD were older than 60 years, the linear hyperintensity might have been the result of nonspecific changes in the aging brain, though it is possible that nigrostriatal degeneration, as the main patho- 
logic feature of PD, contributes to the presence of the linear hyperintensity.

With regard to the selection of the sequences on MR imaging, we evaluated the findings only on fast spin-echo T2WI and could not make a comparison between fast spin-echo and gradient-echo T2WI or between fast spin-echo T2WI and fluid-attenuated inversion recovery (FLAIR) images. We speculate that fast spin-echo T2WI is more suitable for depicting the linear hyperintensity than gradient-echo T2WI because a susceptibility effect in the GPi on gradient-echo T2WI may cause obscure hyperintensity along the GPi. FLAIR images may be inferior because of their low signal-to-noise ratio compared with fast spin-echo T2WI.

MR imaging features of MJD have been reported to be atrophy of the pons, especially pontine tegmentum; atrophy of the cerebellum; fourth ventricular dilation; atrophy of the superior cerebellar peduncles; and mild frontal atrophy. ${ }^{13,14}$ Tokumaru et $\mathrm{al}^{13}$ failed to show atrophy of the globus pallidus, and they did not mention any abnormalities of signal intensity surrounding the GPi. The MR imaging diagnosis of MJD is mainly based on loss of volume of the cerebellum and pons; there is no confirmed change in signal intensity to suggest the diagnosis of MJD. In patients with clinically suspected MJD, a finding of linear hyperintensity along the medial margin of the GPi can be useful for further confirmation of the diagnosis.

\section{Conclusion}

Linear hyperintensity along the medial margin of the GPi on T2WI reflects some degeneration in patients with MJD, and it is highly suggestive of MJD in younger people. However, it also appears in healthy older subjects, and it is important to keep in mind that the hyperintensity is highly suggestive of, but not specific for, MJD in older people.

\section{References}

1. Takiyama Y, Oyanagi S, Kawashima S, et al. A clinical and pathologic study of a large Japanese family with Machado-Joseph disease tightly linked to the DNA markers on chromosome 14q. Neurology 1994;44:1302-08

2. Yamada S, Nishimiya J, Nakajima T, et al. Linear high intensity area along the medial margin of the internal segment of the globus pallidus in MachadoJoseph disease patients. J Neurol Neurosurg Psychiatry 2005;76:573-75

3. Kawaguchi $Y$, Okamoto $\mathrm{T}$, Taniwaki $\mathrm{M}$, et al. CAG expansions in a novel gene for Machado-Joseph disease at chromosome 14q32.1. Nat Genet 1994;8:221-28

4. Litvan I, Bhatia KP, Burn DJ, et al. Movement Disorders Society Scientific Issues Committee report: SIC task force appraisal of clinical diagnostic criteria for Parkinsonian disorders. Mov Disord 2003;18:467-86

5. Carpenter MB. Corpus striatum and related nuclei. In: Core text of neuroanat omy. 4th ed. Baltimore: Williams and Wilkins; 1996:325-60

6. Iwabuchi K, Yagishita S. A neuropathological study on autosomal dominant forms of spinocerebellar degeneration. [in Japanese] Neuropathology 1991;11:155-77

7. Yuasa T, Ohama E, Harayama H, et al. Joseph's disease: clinical and pathological studies in a Japanese family. Ann Neurol 1986;19:152-57

8. Lavoie B, Smith Y, Parent A. Dopaminergic innervation of the basal ganglia in the squirrel monkey as revealed by tyrosine hydroxylase immunohistochemistry. J Comp Neurol 1989;289:36-52

9. Parent A, Côté PY, Lavoie B. Chemical anatomy of primate basal ganglia. Prog Neurobiol 1995;46:131-97

10. Gunning-Dixon FM, Head D, McQuain J, et al. Differential aging of the human striatum: a prospective MR imaging study. AJNR Am J Neuroradiol 1998;19:1501-07

11. Raz N, Rodrigue KM, Kennedy KM, et al. Differential aging of the human striatum: longitudinal evidence. AJNR Am J Neuroradiol 2003;24:1849-56

12. Walhovd KB, Fjell AM, Reinvang I, et al. Effects of age on volumes of cortex, white matter and subcortical structures. Neurobiol Aging 2005;26:1261-70; discussion $1275-78$

13. Tokumaru AM, Kamakura K, Maki T, et al. Magnetic resonance imaging findings of Machado-Joseph disease: histopathologic correlation. J Comput Assist Tomogr 2003;27:241-48

14. Yoshizawa T, Watanabe M, Frusho K, et al. Magnetic resonance imaging demonstrates differential atrophy of pontine base and tegmentum in MachadoJoseph disease. J Neurol Sci 2003;215:45-50 